Letter to the Editor

\title{
Revisiting amlodipine induced pedal edema: a student's perspective
}

Sir,

Amlodipine, a dihydropyridine, is used frequently used in the treatment of hypertension and angina pectoris. The drug acts by blocking the L-gated calcium channels which causes the blood vessels to relax. It's taken orally and excreted through the urine like many other drugs. ${ }^{1}$ Calcium channel blockers are usually well tolerated but as always exceptions always exist. Common side effects are constipation, dizziness, flushing, and pre-tibial edema. Pre-tibial edema has been a very common side effect that has lead the drug to be withdrawn in some patients therapy. ${ }^{2}$

When data was collected at the Kasturba Medical Hospital at Manipal University in Karnataka, amlodipine induced pedal edema was common in about $15.6 \%$ of the patients. Amlodipine causes arteriolar dilation and has no effect on the veins. As arteries are dilated, more blood reaches the veins and this leads too pooling of the blood. Veins don't have any muscular activity so all the excess blood enters the interstitial space leading to pedal edema. ${ }^{3}$

Table 1: Amlodipine induced pedal edema: patient characteristics.

\begin{tabular}{|lll|l|}
\hline Variables & & Males & Females \\
\hline No. of patients & 50 & $26(52 \%)$ & $24(48 \%)$ \\
\hline Average age (years) & 50.06 & 59.11 & 40.58 \\
\hline Average dose (mg) & 4 & 5 & 3.5 \\
\hline $\begin{array}{l}\text { No. of patients with } \\
\text { pedal edema }\end{array}$ & 7 & $5(10 \%)$ & $2(4 \%)$ \\
\hline
\end{tabular}

The data that was collected over a two month proves that both dose and duration of drug therapy play a role in the cause of edema.

A study comparing the efficacy and safety of amlodipine and felodipine by Milind Pardeshi and SV Dange ${ }^{4}$ shows that about $11 \%$ of the patients had amlodipine induced pedal edema. Hence they were able to conclude that amlodipine was more likely to cause pedal edema. So it may be safe to say that about one in every ten patients encounter amlodipine induced pedal edema. Most of the time the doctor reduced the dose of amlodipine to see if the pedal edema got better, since pedal edema is dose dependent. If the dose is already too low then the drug could be withdrawn.
At the Kasturba Medical Hospital in Manipal, age and sex seemed to have a correlation with the side effect. Patients in their late fifties onwards tend to have pedal edema more. This might be due to the fact that the valves in the veins aren't as efficient anymore and this can lead to excess pooling of the blood. Also, males tended to develop pedal edema more than females. Due to the fact that a lot of these patients were at the lower level on the weighing scale, even a tiny bit of blood pooling might look like a bigger deal that it actually is.

Sometimes the side effect can be dependent on the duration of drug therapy. A patient who's been on amlodipine for more than four years is more likely to develop pedal edema rather than a patient on amlodipine with the same dose for only one year. ${ }^{5}$

In another study by Walid Nuri, and Juan Carlos Pelayo, one of the subjects had to be removed from the amlodipine therapy due to the pedal edema. In such cases when the drug is not well tolerated, patients are switched to other alternatives such as nifedipine, felodipine, and verapamil.

Amlodipine is a very common drug used in the cardiovascular department in the management of blood pressure and hypertension regardless of its side effects. Some studies indicate that the pedal edema can be offset with combination therapy, such as amlodipine with benazepril. $^{7}$ Age, weight, drug dose might all play a role in the cause of the side effect but they don't outweigh the benefits of the drug.

\section{Swathi Nemani ${ }^{1}$, Yeshwanth Rao $^{2 *}$}

Student-Alumni, Kasturba Medical College-International Center (KMC-IC), Manipal, India

Professor \& Course Director, Department of

Pharmacology, Kasturba Medical College-International Center (KMC-IC), Manipal, India

*Correspondence to: Dr. Yeshwanth Rao, Email: yashwanthrao2000@gmail.com

\section{REFERENCES}

1. Clavijo GA, de Clavijo IV, Weart CW. Amlodipine: a new calcium antagonist. Am J Hosp Pharm 1994:51:59-68 
2. Sica DA. Calcium Channel Blocker-Related Peripheral Edema: Can It Be Resolved? J Clin Hypertens (Greenwich) 2003;5:291-4.

3. DeWitt CR, Waksman JC. Pharmacology, Pathophysiology and Management of Calcium Channel Blocker and $\beta$-Blocker Toxicity. Toxicol Rev 2004:23(4):223-38.

4. Pardeshi M, SV Dange SV. Comparison of Efficacy and Safety of Amlodipine and Felodipine-ER in Patients of Essential Hypertension. Bombay Hospital Journal 2004:46(2).
5. Messerli FH, Grossman E. Pedal Edema-Not All Dihydropyridine Calcium Antagonists Are Created Equal. AJH 2002;15:1019-20.

6. Juan Carlos Pelayo, Wahd Nuri. Medical/Statistical Review. P.44.

7. Chrysant SG, Sugimoto DH, et al. The effects of high-dose amlodipine/benazepril combination therapies on blood pressure reduction in patients not adequately controlled with amlodipine monotherapy. Blood Press Suppl 2007;1:10-7.

doi:10.5455/2319-2003.ijbcp20131030

Cite this article as: Nemani S, Rao Y. Revisiting amlodipine induced pedal edema: a student's perspective. Int J Basic Clin Pharmacol 2013;2:661-2. 